\title{
Morphological Alterations in the Kidney of Rats with Natural and Experimental Leptospira Infection
}

\author{
M. Tucunduva de Faria*, D. A. Athanazio ${ }^{\dagger,}$, E. A. Gonçalves Ramos ${ }^{\dagger, \ddagger}$, \\ E. F. Silva ${ }^{\dagger, \S}$, M. G. Reis ${ }^{\dagger, \uparrow}$ and A. I. Ko ${ }^{\dagger, \|}$ \\ * Embrapa Amazônia Oriental, Belém-PA, Brazil, ${ }^{\dagger}$ Gonçalo Moniz Research Center, Oswaldo Cruz Foundation, \\ Ministry of Health, Salvador-BA, Brazil, ${ }^{\circledR}$ Federal University of Bahia, Salvador-BA, Brazil, ${ }^{\S}$ Federal University of Pelotas-RS, Brazil, \\ and "Division of International Medicine and Infectious Disease, Weill Medical College of Cornell University, New York, NY, USA
}

\begin{abstract}
Summary
Leptospirosis is a widespread anthropozoonosis, with a broad array of mammalian reservoirs, occurring as rural endemics, urban outbreaks related to floods, and emergent disease associated with water sports and recreational exposure in developed countries. Rats are the major source of human infection, particularly in urban areas; however few reports have focused on the pathology of leptospirosis in this host. This study reports pathological changes in 60 kidneys from captured wild rats and compares these with changes in the kidney of Wistar rats experimentally infected with Leptospira interrogans serovar Copenhageni strain FIOCRUZ L1-130. A broad range of morphological alterations were detected in the kidneys from captured rats but interstitial nephritis was the only feature reproduced under experimental conditions. The role of interstitial nephritis in the pathogenesis of leptospirosis is reviewed and it is suggested that rats may provide a potential tool for the study of colonization mechanisms and host resistance in acute leptospiral disease.
\end{abstract}

(C) 2007 Elsevier Ltd. All rights reserved.

Keywords: leptospirosis; Rattus norvegicus; interstitial nephritis; kidney

\section{Introduction}

Leptospirosis is a widespread anthropozoonosis caused by pathogenic spirochaetes of the genus Leptospira. The infection may be transmitted to humans by direct contact or indirect exposure to urine from mammalian hosts such as peri-domiciliary rodents and farm, wild and domestic animals. The broad range of mammalian reservoirs explains a diverse array of epidemiological contexts such as rural endemics, urban outbreaks related to rainy seasons and floods, and emergent disease related to water sports and recreational exposure in developed countries. The most common presentation of human infection is oligosymptomatic or an undifferentiated febrile illness. The major impact of leptospirosis is associated with severe forms of disease including:

Correspondence to: M. Tucunduva de Faria, Embrapa Amazônia Oriental, Trav. Enéas Pinheiro S/N, Caixa Postal 48, Belém-PA, 66095-100, Brazil (e-mail: tucun@cpatu.embrapa.br).
Weil's syndrome of jaundice, acute renal failure and haemorrhagic diathesis with an overall mortality of $10-15 \%$ in most reported case series, and severe pulmonary haemorrhage syndrome (SPHS) which frequently has mortality higher than 50\%. In Salvador, Brazil, leptospirosis occurs as epidemics related to the rainy season, floods and rat infestation of homes, with mortality frequently higher than 50\% (McBride et al., 2005).

Rats are a major natural reservoir of infection but are relatively resistant to leptospiral disease. Despite the importance of the rat in disease transmission, few reports have focused on the underlying pathology in this species. A recent study has compared antigenic switching of the leptospiral surface in host species either susceptible (guinea-pig), or resistant to (rat), acute lethal disease (Nally et al., 2005a). Pathological studies of captured wild rats have shown a broad spectrum of lesions which may not necessarily be related to 
leptospires (Laurain, 1955). One investigation of captured urban rats suggested that recent infections, as suggested by high microagglutination test (MAT) titres, were related to inflammatory infiltration of renal tissue (Sterling and Thiermann, 1981). By contrast, when Sprague-Dawley rats were experimentally infected and evaluated 21 days later, no microscopical changes were observed in the kidney (Nally et al., 2005a).

Thus, to date the pathology of leptospiral infection in rats has been poorly defined. Any use of rat models for leptospirosis research depends upon clear understanding of the basic host-pathogen interactions and, surprisingly, no study to date has focused on this issue. The present study reports the renal pathology associated with spontaneously arising leptospiral infection in captured urban rats in Salvador and with experimental infection of Wistar rats. This comparison enables evaluation of the renal histopathology of infected rats under controlled conditions as well as in the urban environment where a diverse array of chemical and biological factors may influence the natural history of infection.

\section{Materials and Methods}

\section{Leptospires Used for Inoculation of Wistar Rats}

Leptospires were cultivated in liquid Ellinghausen and McCullough, modified by Johnson and Harris, liquid medium (EMJH, Difco Laboratories, Detroit, USA) at $28.4{ }^{\circ} \mathrm{C}$ and counted in a Petroff-Hausser counting chamber (Fisher Scientific, Pittsburgh, PA, USA) (Ellinghausen and McCullough, 1962). A low passage L. interrogans serovar Copenhageni strain L1-130 was used. This is a clinical isolate representative of the most important leptospiral serovar and genotype in Salvador (Ko et al., 1999). The lethal dose $50 \%\left(\mathrm{LD}_{50}\right)$ in hamsters was calculated as lower than 100 leptospires in four different experiments. This isolate reproduces acute lethal infection in hamsters characterized pathologically by renal tubular epithelial cell swelling and haemorrhage, while convalescent animals exhibit regenerative tubular changes and moderate to severe interstitial nephritis. The organisms had been passaged and re-isolated from hamsters four times following initial isolation from a blood culture of a patient with leptospirosis. Aliquots of this strain were stored at $-70^{\circ} \mathrm{C}$. Frozen aliquots were thawed and passaged in liquid medium seven times prior to being used as a low-passagenumber isolate in the present infection experiments.

\section{Experimental Infection of Wistar Rats}

Four- to five-week-old Wistar rats were inoculated intraperitoneally with $10^{8}$ spirochaetes and sacrificed at determined intervals up to four months post-infection. The dose of $10^{8}$ leptospires was selected, as in pilot studies doses of $10^{4}$ leptospires or lower failed to induce reproducible renal colonization. In one other published study that used the same strain for experimental infection of guinea-pigs and rats (Nally et al., 2005a), inoculation doses were arbitrarily defined as $10^{5}$ for guineapigs and $10^{7}$ for Sprague-Dawley rats. Control animals received $1 \mathrm{ml}$ of sterile EMJH medium by intraperitoneal injection. During necropsy, urine was aspirated from the bladder under aseptic conditions for culture. The right kidney was removed and macerated inside a sterile syringe for isolation in culture medium. The left kidney was removed and cut longitudinally before being fixed in neutral-buffered formalin. These samples were paraffin wax-embedded and tissue sections were prepared for staining by haematoxylin and eosin (HE) and Warthin-Starry silver impregnation (Faine, 1965, 1982).

\section{Captured Animals}

Rats (brown rat) were captured in several neighbourhoods of Salvador; nine of which had reported human cases of severe leptospirosis and two without reported cases. Captures were made between May 1998 and March 1999. Animals were trapped in $20 \times 20 \times$ $60 \mathrm{~cm}$ Tomahawk ${ }^{\circledR}$ cages - near open sewers in the vicinity of houses from which human cases of severe leptospirosis had been reported. Captured rats were necropsied after 1-24h. Kidney and urine samples were collected as previously described for experimentally infected rats.

\section{Leptospira Isolation in Culture Medium}

Four drops of urine were immediately inoculated into four different tubes containing EMJH medium. The renal macerate was first inoculated into an EMJH tube for $30 \mathrm{~min}$ to allow sedimentation of tissue debris. Following this step, $0.5 \mathrm{ml}$ of supernatant was inoculated into each of four new liquid EMJH tubes. All eight EMJH culture tubes were maintained in an incubator at $28.4^{\circ} \mathrm{C}$. Cultures were evaluated weekly by dark field microscopy for 8 weeks or until leptospires were observed.

\section{Renal Histopathology}

Samples of kidney from captured rats were fixed in neutral-buffered formalin and embedded in paraffin wax. Tissue sections $(2 \mu \mathrm{m})$ were stained by HE, periodic acid-Schiff (PAS) methenamine silver (PAS-M) and Azan Mallory. From 107 captured brown rats, all 15 culture-negative and 45 out of 92 culture-positive kidneys were selected for morphological analysis. 
Culture-positive cases were randomly selected in proportion to the total of number of rats captured in each neighbourhood. Microscopical lesions were classified according to previously reported criteria (Pirani et al., 1964; Bernstein and Chrurg, 1992).

\section{Indirect Immunofluorescence}

Frozen sections $(4-5 \mu \mathrm{m})$ were thawed to room temperature and incubated with primary polyclonal antibody prepared by immunizing New Zealand white rabbits with whole cell antigens from $L$. interrogans serovar Icterohaemorrhagiae strain RGA (Faine, 1982). After three rinses in phosphate buffered saline (PBS; $\mathrm{pH} 7.2,0.01 \mathrm{M})$, the samples were incubated with secondary antibody consisting of goat-derived anti-rabbit IgG conjugated with fluorescein isothiocyanate (FITC) (Jackson Immunoresearch, West Grove, PA, USA).

\section{Statistical Analysis}

For data from captured rats, the chi-square and Fischer exact tests, with 95\% significance level, and the kappa index were used to compare results using EPI INFO ${ }^{\circledR}$ software.

\section{Results}

\section{Experimentally Infected Rats}

A total of six independent experiments were performed using 4- to 5-week-old Wistar rats. The intervals from infection to necropsy examination ranged from 1 week to 4 months. All animals had detectable leptospires in renal tissue by culture re-isolation, Warthin-Starry stain (Fig. 1), indirect immunofluorescence or a combination of these methods. Interstitial nephritis was the only morphological alteration observed in these rats. No lesions were detected in liver, lungs, heart, spleen, brain, skeletal muscle, skin or eyes.

The frequency of interstitial nephritis in experimentally infected rats is summarized in Table 1 . Among the 24 rats examined, only one female animal had detectable inflammatory infiltrates in the first 4 weeks postinfection. The lesion was detected more often in animals infected for periods of 1 month or more: $2 / 8$ $(25 \%)$ males and $5 / 13(38 \%)$ females at 1 month, $1 / 2$ $(50 \%)$ males and $4 / 5(80 \%)$ females at 2 months, $2 / 2$ $(100 \%)$ males and $3 / 5(60 \%)$ females at 3 months, and $1 / 5(20 \%)$ females at 4 months. All cases had a similar pattern of focal mild infiltration of lymphocytes, macrophages and plasma cells surrounding small cortical arteries (Fig. 2A-C). In a single female rat examined 2 months post-infection there were small foci of granulomatous inflammation in the renal cortex.

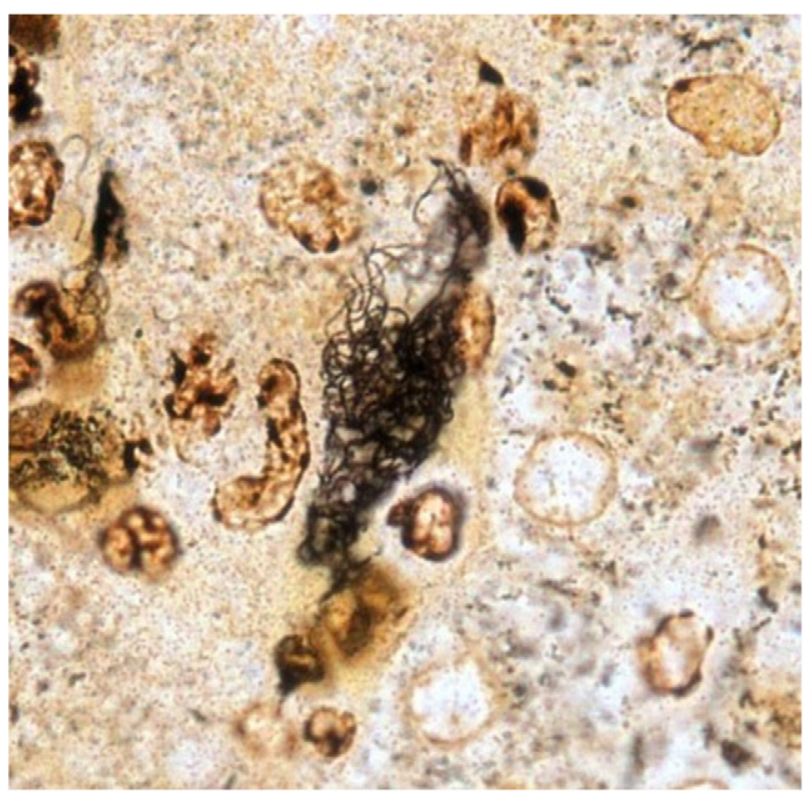

Fig. 1. Dense colonization of renal tubules by leptospires in experimentally infected rats 4 weeks post-infection. Warthin-Starry silver stain. $\times 2000$.

Table 1

Histopathological changes in the kidneys of rats experimentally infected with $L$. interrogans serovar Copenhageni strain L1-130

\begin{tabular}{lc}
\hline Time post-infection & Interstitial nephritis $(n=(\%))$ \\
\hline 1 week & $1 / 8(12.5)$ \\
2 weeks & $0 / 8(0)$ \\
3 weeks & $0 / 8(0)$ \\
4 weeks & $7 / 21(33.3)$ \\
2 months & $5 / 7(71.4)$ \\
3 months & $5 / 7(71.4)$ \\
4 months & $1 / 7(14.3)$ \\
\hline
\end{tabular}

The results presented are derived from six independent experiments. For each time point there were 2-8 uninfected controls in which there were no pathological changes.

These foci were characterized by the presence of multinucleate giant cells that were surrounded by lymphocytes, suggesting an immune-mediated reaction (Fig. 2E and F). No control animals inoculated with EMJH medium developed renal inflammatory changes.

\section{Captured Rats}

All 60 captured rats had normal external appearance and were apparently healthy. They did not exhibit any external parasites, had normal pale red mucosae, and showed aggressive behaviour. Some animals had scars on their feet. In all cases there were morphological alterations in renal tissue and these are summarized in Table 2. 

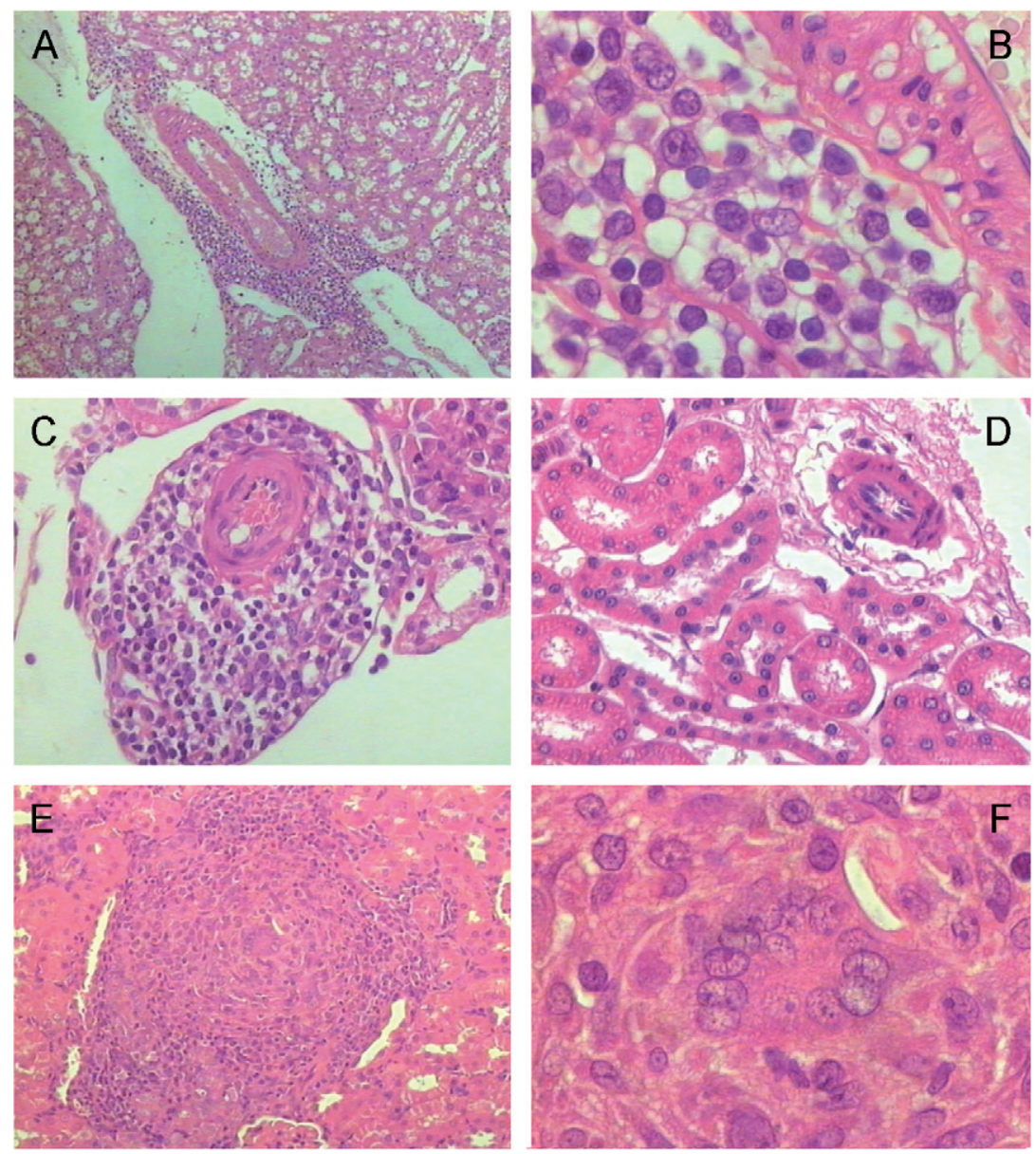

Fig. 2. Histopathological changes in the kidneys of rats inoculated with 108 Leptospira interrogans serovar Copenhageni strain FIOCRUZ L1-130. Rats were sacrificed 1 month $\left(\mathrm{A}^{-} \mathrm{C}\right)$ and 2 months $(\mathrm{E}$ and $\mathrm{F})$ post-infection. (A) The most common lesion in the kidney of chronic carrier rats is periarteriolar infiltration with minimal injury of adjacent proximal tubules. HE. $\times 200$. (B) A higher magnification allows identification of histiocytes and lymphocytes surrounding the vessel. HE. $\times 1000$. (C) In another example, the infiltrate is observed surrounding a cortical arteriole. HE. $\times$ 400. (D) In contrast, non-infected control rats exhibited normal renal tissue without inflammatory foci. HE. $\times$ 400. $(\mathrm{E})$ and $(\mathrm{F})$ In one female rat granulomatous inflammation was observed 2 months postinfection. HE. $(\mathrm{E}) \times 200 .(\mathrm{F}) \times 1000$.

Multifocal calcification was observed in $22 \%$ of culture-positive and $20 \%$ of culture-negative rats (Fig. 3A). Glomerulonephritis was detected in $7 \%$ of culture-positive and $20 \%$ of culture-negative animals. In all cases, glomerulonephritis was classified as mesangioproliferative with segmental and focal distribution. No protein deposits were detected by the AZAN stain. Minimal glomerular alterations were defined by the presence of mild thickening of the mesangial matrix, parietal epithelial cell swelling and a normal number of glomerular cells. These alterations were detected in $33 \%$ of culture-positive and $20 \%$ of culturenegative rats (Fig. 3B). The most common histological change detected in all groups was the presence of tubular epithelial hyaline droplets, observed in $64 \%$ of culture-positive and $87 \%$ of culture-negative animals (Fig. 3C).
Three lesions described as typical of renal leptospirosis were observed. Interstitial nephritis was the most common, being detected in $25(56 \%)$ of culture-positive and $8(53 \%)$ of culture-negative rats (Fig. 3D). Tubular regeneration was observed in $9(20 \%)$ of culture-positive and 2 $(13 \%)$ of culture-negative animals, while tubular necrosis (Fig. 3E) was found in only $2(4 \%)$ of culture-positive and $1(7 \%)$ of culture-negative rats. The frequencies of all detected lesions were not significantly different when compared according to urine or kidney culture status (Table 2). In two cases filarial parasites were detected in renal tissue, localized to the pelvis and calices.

\section{Discussion}

An important tool for basic research on infectious diseases is the application of models of natural resistance 
Table 2

Histopathological changes in the kidneys of 60 captured wild urban rats divided according to Leptospira spp. culture status

\begin{tabular}{lcc}
\hline Lesion type & \multicolumn{2}{c}{$n=(\%)$} \\
\cline { 2 - 3 } & $\begin{array}{c}\text { Culture-positive } \\
(n=45)\end{array}$ & $\begin{array}{c}\text { Culture-negative } \\
(n=15)\end{array}$ \\
\hline Tubular epithelial hyaline droplets & $29(64)$ & $13(87)$ \\
Glomerular and cortical tubular & $27(60)$ & $5(33)$ \\
proteinuria & & \\
Medullary proteinuria/tubular & $25(56)$ & $9(60)$ \\
dilation & $25(56)$ & $8(53)$ \\
Chronic interstitial nephritis & $15(33)$ & $3(20)$ \\
Minimal glomerular alterations & $10(22)$ & $3(20)$ \\
Multifocal calcification & $9(20)$ & $2(13)$ \\
Tubular regeneration & $7(16)$ & $1(07)$ \\
Chronic pyelitis & $5(11)$ & $1(06)$ \\
Interstitial fibrosis & $3(07)$ & $3(20)$ \\
Proliferative mesangial & & \\
glomerulonephritis & $2(04)$ & $1(07)$ \\
Tubular necrosis & $2(04)$ & $0(0)$ \\
Acute pyelonephritis/abscesses & $2(04)$ & $0(0)$ \\
Parasites &
\end{tabular}

and susceptibility to disease as exemplified by the use of different strains of mouse to reproduce distinct forms of leishmaniosis (Barral-Netto et al., 1987; Falcoff et al., 1991). Most publications on experimental leptospirosis have focused on models of acute lethal infection in guinea-pigs, hamsters and some strains of mouse (Miller et al., 1974; Oliva et al., 1994; Nally et al., 2004, 2005a, b; Viriyakosol et al., 2006). Mouse models have been recently used to explore genetic susceptibility to severe leptospirosis (Nally et al., 2005b; Viriyakosol et al., 2006); however mice are not considered an ideal model, since variations in inoculation dose, mouse strain and leptospiral serovars has led to variable results (Faine, 1962; McBride et al., 2005). Rats are theoretically an ideal model to explore natural resistance to disease since they are consistently reported to have an asymptomatic carrier state (Bertok et al., 1964; Thiermann, 1981; Natarajaseenivasan and Ratnam, 1997). Recent interest has re-emerged in employing the rat model for comparison of the difference in leptospiral antigen expression in models of acute disease and persistent carriage (Nally et al., 2005a). Surprisingly, few limited evaluations of pathological findings are available for naturally and experimentally infected rats.

An important question for experimental purposes is related to the ability of laboratory infection to reproduce the host-pathogen interaction that occurs in natural infection. The pathogenesis of natural leptospiral infection in rats has not been extensively studied, and is complicated by a wide array of potentially confusing environmental factors that may also induce pathology in urban wild rats. To our knowledge, only two previous studies have evaluated renal morphology in captured urban rats carrying leptospires. The first of these included a series of 11 rats captured in Denver, USA, in which 4 cases of interstitial nephritis were reported (one with proven leptospiral infection), and a broad spectrum of other pathological changes which could not be attributed to leptospires (Laurain, 1955). In the second study, an electron microscopical investigation (with no light microscopical evaluation described) was performed with tissue from six urban rats captured in Detroit, USA, that were selected by high MAT titres. This investigation revealed interstitial oedema and infiltration with plasma cells, lymphocytes and histiocytes (Sterling and Thiermann, 1981). The authors suggested that MAT titres should reflect recent infection so this interstitial nephritis may represent a transient subclinical pathology that could rapidly be reversed. This interpretation is controversial, since there is a clear dissociation between active infection detected by microbiological methods and serum antibody titres in captured animals (Sunbul et al., 2001).

In the present study, we evaluated renal pathology in urban rats from a city where the prevalence of leptospiral isolation by urine/kidney culture was $82 \%$. We have shown that, even in a population with a high rate of leptospiral infection, the finding of interstitial nephritis cannot be attributed to leptospires since the frequency of inflammation is comparable in animals with and without active infection. Thus, a wide array of noxious environmental exposures resulting in background pathological change means that it is impossible to accurately evaluate natural infection outcomes in captured rats.

In contrast, our experimental findings showed that interstitial nephritis may occur at one month or later after infection. A total of $43 \%$ of all infected rats sacrificed between one and 4 months developed focal inflammatory renal disease. To our knowledge, the single report of renal pathology in experimentally infected rats is from a recent study which used Sprague-Dawley rats infected with a human isolate of $L$. interrogans serovar Copenhageni. In a brief description, the authors did not report any histopathological changes 21 days post-infection (Nally et al., 2005a). Our results are consistent with this finding since no animal sacrificed after 3 weeks had evidence of inflammation. Ongoing experiments in a Wistar rat model suggest that renal colonization is established around the seventh day after infection and this process is not related to any microscopical lesion (data not shown). We speculate that renal inflammatory infiltrates are a late feature of leptospiral infection in rats. This morphological picture associated with subclinical persistent colonization places the rat model close to the natural behaviour of 

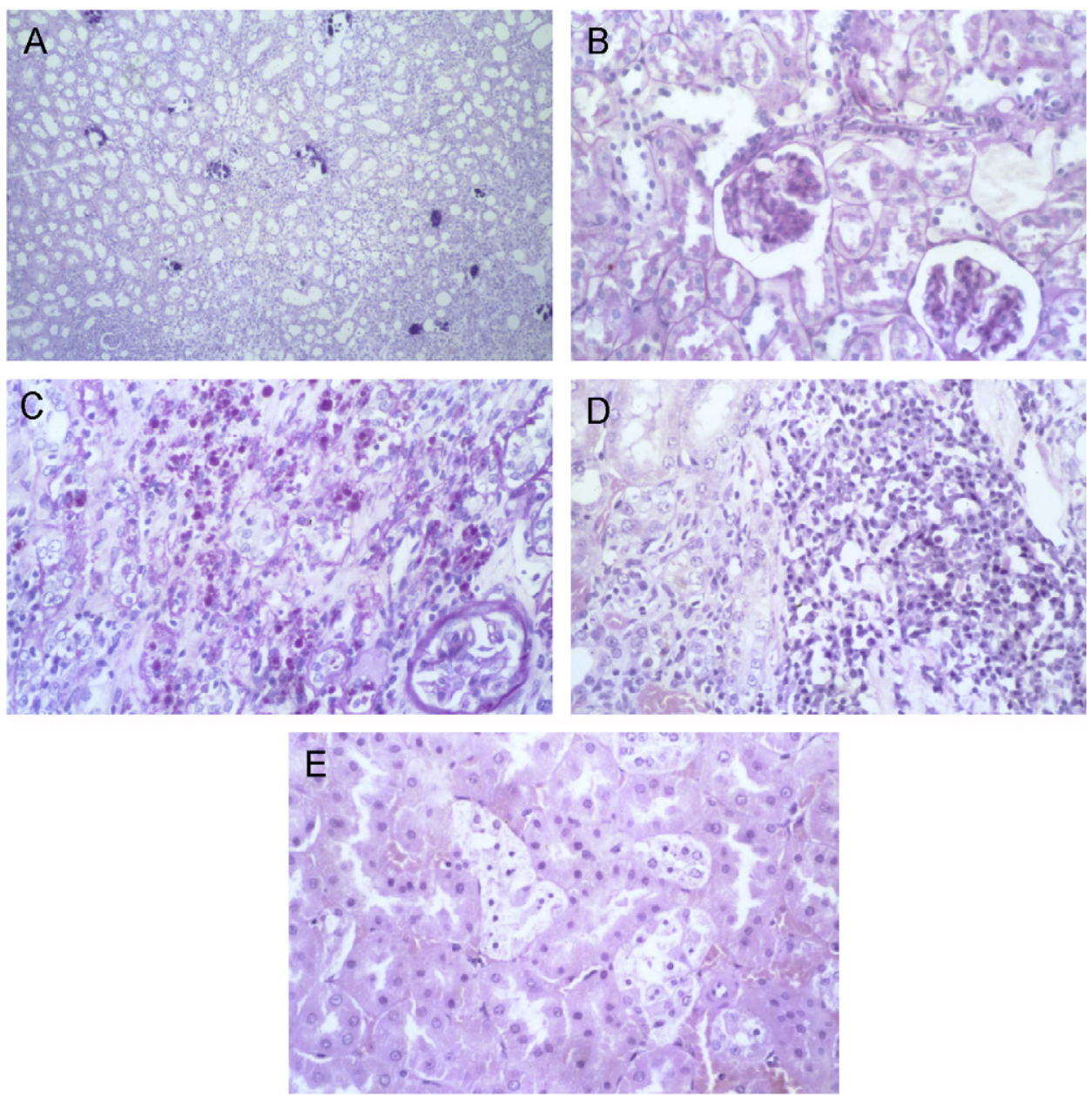

Fig. 3. Histopathological changes in the kidneys of wild captured rats. (A) Multiple foci of calcification visualized as basophilic deposits in medullary tissue. HE. $\times$ 100. (B) Mesangial thickening in two glomeruli. HE. $\times 200 .($ C) Tubular epithelial hyaline droplets in cortical tissue. PAS. $\times$ 200. (D) Foci of interstitial nephritis. HE. $\times 200$. (E) Tubular necrosis visualized as areas of marked epithelial cell swelling with pyknotic nuclei. HE. $\times 400$.

leptospiral infection in cattle and pigs (Tabel and Karstad, 1967; Yener and Keles, 2001; Boqvist et al., 2003).

A single case of interstitial nephritis was associated with the development of renal microgranulomata. Although the low frequency of this lesion type must be interpreted cautiously, it is relevant to review the role of cellular immunity and granulomatous inflammation in leptospirosis. Recent data implicate strong cellular immune responses in protecting cattle against serovar Hardjo (Naiman et al., 2001, 2002; Brown et al., 2003). Multinucleate giant cells have been reported in the kidneys of naturally infected cattle (Hadlow and Stoenner, 1955; Burdin, 1963; Amatredjo et al., 1976; Yener and Keles, 2001). Although some authors have implicated a foreign body reaction against degenerative tubules or their content, the recent observation of leptospiral antigen in those giant cells favours the interpretation that these findings reflect a true response to persistent colonization (Yener and Keles, 2001). Multinucleate giant cells have also been detected in the liver of vaccinated dogs experimentally infected with pathogenic leptospires, although these were reported to be associated with haemosiderin deposits (Adamus et al., 1997).

In summary, the present study reports a histopathological analysis of kidney tissues from wild captured and experimentally infected rats. Interstitial nephritis was the only lesion attributable to leptospiral infection. We suggest that rats provide a potential experimental tool to understanding mechanisms of resistance to acute disease and renal colonization. However, it should be noted that complete resistance to clinical disease is frequently associated with underlying subclinical inflammatory lesions.

\section{Acknowledgements}

This work was supported by the Brazilian National Research Council (grants 300861/1996, 554788/2006) and 
the National Institutes of Health (grants AI052473 and TW00919).

\section{References}

Adamus, C., Buggin-Daubie, M., Izembart, A., SonrierPierre, C., Guigand, L., Masson, M. T., Andre-Fontaine, G. and Wyers, M. (1997). Chronic hepatitis associated with leptospiral infection in vaccinated beagles. Fournal of Comparative Pathology, 117, 311-328.

Amatredjo, A., Campbell, R. S. and Trueman, K. F. (1976). A study of nephritis of beef cattle in North Queensland. Australian Veterinary fournal, 52, 398-402.

Barral-Netto, M., Cardoso, S. A. and Barral, A. (1987). Different patterns of disease in two inbred mouse strains infected with a clone of Leishmania mexicana amazonensis. Acta Tropica, 44, 5-11.

Bernstein, J. and Chrurg, J. (1992). Urinary Tract Pathology: An Illustrated Practical Guide to Diagnosis, Raven Press, New York.

Bertok, L., Kemenes, F. and Simon, G. (1964). Fatal Leptospirosis Icterohaemorrhagica induced by ethionine in albino rats. Fournal of Pathological Bacteriology, 88, 329-331.

Boqvist, S., Montgomery, J. M., Hurst, M., Thu, H. T., Engvall, E. O., Gunnarsson, A. and Magnusson, U. (2003). Leptospira in slaughtered fattening pigs in southern Vietnam: presence of the bacteria in the kidneys and association with morphological findings. Veterinary Microbiology, 93, 361-368.

Brown, R. A., Blumerman, S., Gay, C., Bolin, C., Duby, R. and Baldwin, C. L. (2003). Comparison of three different leptospiral vaccines for induction of a type 1 immune response to Leptospira borgpetersenii serovar Hardjo. Vaccine, 21, 4448-4458.

Burdin, M. L. (1963). Renal histopathology of leptospirosis caused by Leptospira grippotyphosa in farm animals in Kenya. Research in Veterinary Science, 4, 423-429.

Ellinghausen, H. G. and McCullough, W. G. (1962). Nutrition of Leptospira pomona and growth of 13 other serotypes: fractionation of oleic albumin complex and a medium of bovine albumin and polysorbate 80. American fournal of Veterinary Research, 26, 45-51.

Faine, S. (1962). The growth of Leptospira australis B in the kidneys of mice in the incipient experimental carrier state. Journal of Hygiene (Cambridge), 60, 435-442.

Faine, S. (1965). Silver staining of spirochaetes in single tissue sections. Fournal of Clinical Pathology, 18, 381-382.

Faine, S., Ed. (1982). Guidelines for the Control of Leptospirosis. World Health Organization, Geneva.

Falcoff, R., Barral-Netto, M., Stiffel, C., Bouthillier, Y., Barral, A., Freitas, L. A., Mevel, J. C. and Mouton, D. (1991). Variations in susceptibility to Leishmania amazonensis infection in lines of mice selected for high or low immunoresponsiveness. Parasite Immunology, 13, 639-647.

Hadlow, W. J. and Stoenner, H. G. (1955). Histopathological findings in cows naturally infected with Leptospira pomona. American fournal of Veterinary Research, 16, 45-56.
Ko, A. I., Galvao Reis, M., Ribeiro Dourado, C. M., Johnson, W. D. and Riley, L. W. (1999). Urban epidemic of severe leptospirosis in Brazil. Salvador Leptospirosis Study Group. Lancet, 354, 820-825.

Laurain, A. R. (1955). Lesions of skeletal muscle in leptospirosis; review of reports and an experimental study. American Journal of Pathology, 31, 501-519.

McBride, A. J., Athanazio, D. A., Reis, M. G. and Ko, A. I. (2005). Leptospirosis. Current Opinion in Infectious Disease, 18, 376-386.

Miller, N. G., Allen, J. E. and Wilson, R. B. (1974). The pathogenesis of hemorrhage in the lung of the hamster during acute leptospirosis. Medical Microbiology and Immunology, 160, 269-780.

Naiman, B. M., Alt, D., Bolin, C. A., Zuerner, R. and Baldwin, C. L. (2001). Protective killed Leptospira borgpetersenii vaccine induces potent Thl immunity comprising responses by CD4 and gammadelta T lymphocytes. Infection and Immunity, 69, 7550-7558.

Naiman, B. M., Blumerman, S., Alt, D., Bolin, C. A., Brown, R., Zuerner, R. and Baldwin, C. L. (2002). Evaluation of type 1 immune response in naive and vaccinated animals following challenge with Leptospira borgpetersenii serovar Hardjo: involvement of $\mathrm{WCl}(+)$ gammadelta and CD4 T cells. Infection and Immunity, 70, 6147-6157.

Nally, J. E., Chantranuwat, C., Wu, X. Y., Fishbein, M. C., Pereira, M. M., Da Silva, J. J., Blanco, D. R. and Lovett, M. A. (2004). Alveolar septal deposition of immunoglobulin and complement parallels pulmonary hemorrhage in a guinea pig model of severe pulmonary leptospirosis. American fournal of Pathology, 164, 1115-1127.

Nally, J. E., Chow, E., Fishbein, M. C., Blanco, D. R. and Lovett, M. A. (2005a). Changes in lipopolysaccharide O antigen distinguish acute versus chronic Leptospira interrogans infections. Infection and Immunity, 73, 3251-3260.

Nally, J. E., Fishbein, M. C., Blanco, D. R. and Lovett, M. A. (2005b). Lethal infection of $\mathrm{C} 3 \mathrm{H} / \mathrm{HeJ}$ and $\mathrm{C} 3 \mathrm{H} / \mathrm{SCID}$ mice with an isolate of Leptospira interrogans serovar Copenhageni. Infection and Immunity, 73, 7014-7017.

Natarajaseenivasan, K. and Ratnam, S. (1997). Experimental leptospirosis in laboratory mice and rats. Fournal of Communicable Disease, 29, 291-293.

Oliva, R., Infante, J. F., Gonzalez, M., Perez, V., Sifontes, S., Marrero, O., Valdes, Y., Farinas, M., Estevez, L. and Gonzalez, I. (1994). Pathologic-clinical characterization of leptospirosis in a golden Syrion hamster model. Archives of Medical Research, 25, 165-170.

Pirani, C. L., Pollak, V. E. and Schwartz, F. D. (1964). The reproducibility of semiquantitative analyses of renal histology. Nephron, 29, 230-237.

Sterling, C. R. and Thiermann, A. B. (1981). Urban rats as chronic carriers of leptospirosis: an ultrastructural investigation. Veterinary Pathology, 18, 628-637.

Sunbul, M., Esen, S., Leblebicioglu, H., Hokelek, M., Pekbay, A. and Eroglu, C. (2001). Rattus norvegicus acting as reservoir of Leptospira interrogans in the middle Black Sea region of Turkey, as evidenced by PCR and presence of serum antibodies to Leptospira strain. Scandinavian fournal of Infectious Diseases, 33, 896-898. 
Tabel, H. and Karstad, L. (1967). The renal carrier state of experimental Leptospira pomona infections in skunks $(M e-$ phitis mephitis). American fournal of Epidemiology, 85, 9-16.

Thiermann, A. B. (1981). The Norway rat as a selective chronic carrier of Leptospira icterohaemorrhagiae. Fournal of Wildlife Disease, 17, 39-43.

Viriyakosol, S., Matthias, M. A., Swancutt, M. A., Kirkland, T. N. and Vinetz, J. M. (2006). Toll-like receptor 4 protects against lethal Leptospira interrogans serovar Icterohaemorrha- giae infection and contributes to in vivo control of leptospiral burden. Infection and Immunity, 74, 887-895.

Yener, Z. and Keles, H. (2001). Immunoperoxidase and histopathological examinations of leptospiral nephritis in cattle. Fournal of Veterinary Medicine Series A, 48, 441-447.

$\left[\begin{array}{c}\text { Received, Fuly 28th, } 2006 \\ \text { Accepted, August 3rd, } 2007\end{array}\right]$ 